\title{
Clinical Application of CSC Telescopic Denture with Magnetic Attachment in Treating Class III Molar Furcation Involvement-A Case Report
}

\author{
Guey-Lin Hou ${ }^{1,2 *}$ \\ ${ }^{1}$ Former Chairman, Dental Department, Chang-Gung Memorial Hospital, Kaohsiung, Taiwan, Republic of China \\ ${ }^{2}$ Former professor, Graduate Institute of Dental Science and Department of Periodontics, Kaohsiung Medical Center, School of Dental Medicine, \\ Kaohsiung Medical University, Kaohsiung, Taiwan, Republic of China
}

*Corresponding author: Former professor, Graduate Institute of Dental Science, Kaohsiung Medical University, Taiwan. Tel: 00886-7-7472606: e-mail: hougl2001@yahoo.com.tw

Received: 31 Aug, 2020 | Accepted: 03 Sep, 2020 | Published: 10 Sep, 2020

Citation: Hou GL (2020) Clinical Application of CSC Telescopic Denture with Magnetic Attachment in Treating Class III Molar Furcation Involvement-A Case Report. Int J Dent Oral Health 6(6): dx.doi.org/10.16966/2378-7090.338

Copyright: (C) 2020 Hou GL. This is an open-access article distributed under the terms of the Creative Commons Attribution License, which permits unrestricted use, distribution, and reproduction in any medium, provided the original author and source are credited.

\begin{abstract}
The clinical observations of periodontal and prosthetic therapies using retrospective and radiographic evaluation have indicated that molars affected with advanced furcation involvement (FI) are more prone to reducing clinical periodontal support and losing clinical attachments. The present case showed approach using molar root separation and the design of unilateral CSCTD and magnetic attachment to treating the right maxillary secondary molar with Class II and III furcation involvement. Result showed a remarkable improvement around periodontal tissue of the inner crowns and infra-furcation and furcation roof 14 years later. It can be concluded that the design of the CSCTD combined with magnetic attachment appears to be an effectiveness method in treating advanced molar furcation problems.
\end{abstract}

Keywords: Molar with FI; CSCTD; Mmagnetic attachment

\section{Introduction}

The clinical observations of periodontal and prosthetic therapies using retrospective and radiographic evaluation have indicated that molars affected with advanced Furcation Involvement (FI) are more prone to reducing clinical periodontal support and losing clinical attachments [1,2]. Predictable and effective treatment of molars affected FI of advanced periodontitis including tunnel preparation, furcation plasty, root sepsrstion, root hemisection, and root amputation these techniques exhibits discrepancies in the success rates and their complications are still controversial [3-8].

However, these procedures have been associated with several complications, problems, such as a residual deeper root concavity, dental plaque control difficulities problems in restoring the resected molars, and root caries $[5,9,10]$. Therefore, how to resolve these complications is the most important using root resection/separation technique and design of periodontal prosthesis fabrication.

The Crown and Sleeve-coping Telescopic Denture (CSCTD) was recommended as a periodontal prosthesis for the treatment of advanced periodontal periodontitis with guided prognosis in clinical evaluation [11,12]. The technique provides some advantages, such as maintaining com- promised abutments, ease of plaque control, a reducing destructive torque force, and less leveling forces on the supported abutments [11-13].

There is little or limited report regarding the treatment of molar with class II and III furcation involvements using CSCTD combined with magnetic attachment. In order to treat Advanced Class II and/ or III molar FI with poor root morphology in the remaining root following root separation and/or resection, we design a modified approach, combination of molar root separation together with a specifically magnetic attachment, name as a "partial CSC telescopic denture with magnetic attachment."

\section{Case Report}

A 66-year-old male in good health, presented with chief complaints of the deterioration of left maxillary second molar with advanced frucation involvement. The symptoms including, gingival bleeding, slight mobility, gingival recession, and inability to chew, had been observed since he was 6 years ago. He had visited some dental clinics been told that the mobile molar with advanced Class II to III should be extracted. Based on the periodontal examination slight gingival recession, heavy deposits of calculus, moderate plaque retention, and Classes II and/or III molar FI were noted on the maxillary $2^{\text {nd }}$ molar. Clinical assessment included GI [14], Pl [15] and alveolar bone loss 
score, Probing Depth (PD), and Clinical Attachment Level (CAL) which were measured from the baseline (2005) until treatment was completed 14 years (2019).

Radiographic illustrated a moderate loss of $\# 17$ on the furcation roof and infra-furcation area of right maxillary $2^{\text {nd }}$ molar. A final diagnosis of moderate adult periodontitis with Classes II and/ or III molar FI was made.

\section{Treatment}

Patient was instructed in thorough plaque control before basic periodontal therapy consisted of basic and routine meticulous subgingival scaling, root planning, and sub-gingival curettage undertaken once a week for 3 months followed by pocket irrigation with chlorhexidine gluconate $0.12 \%$. Supportive periodontal therapy was established every 2 to 4 weeks for 12 months.

The inner crowns with magnetic attachment (\#15) of partial CSC- telescopic denture unit of left maxillary from \#14 to \#17 was constructed after root separation (\#17) (Figure 1). Figure 2 indicates the cross view of the partial CSCTD. Buccal and palatal views of CSCTD with magnetic attachment were showed in Figures 3, and 4. Figure 4 shows the facial view (Figure 5), right posterior view (Figure 6) of inner crowns (\#14,\#15,\#17), and appearance of CSC-telescopic

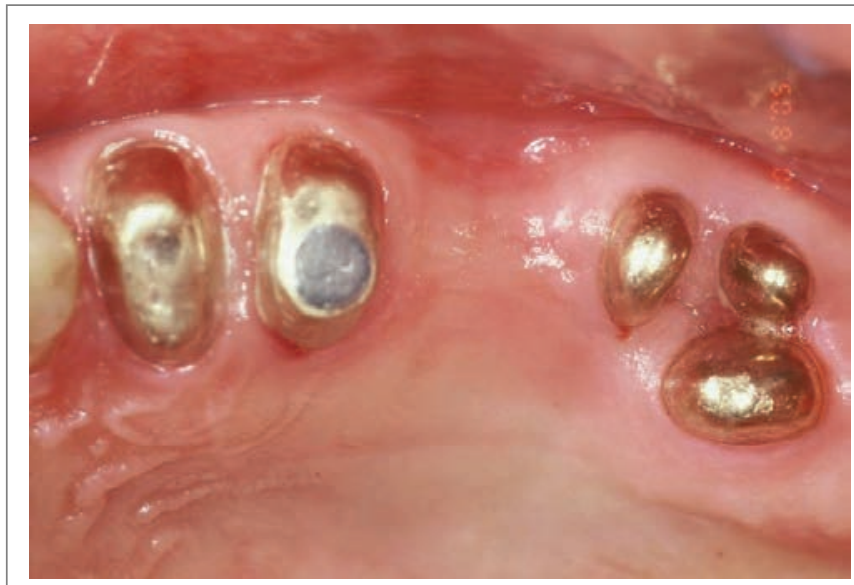

Figure 1: Cross-sectional view shows inner crowns of \# 14, \# 15 with magnetic attachment, and \# 17 with 3 inner crowns after root separation (2006).

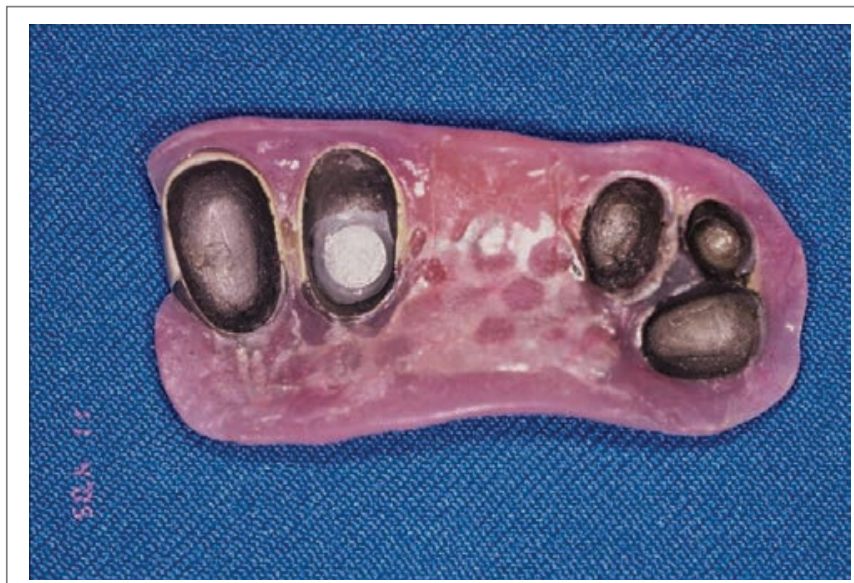

Figure 2: Cross-sectional view of CSC-telescopic crowns with magnetic attachment (\# 14). outer crown of posterior buccal surface (Figure 7).

\section{Discussion}

The primary objectives of CSC telescopic denture with root separation is to preserve the poor abutments with advanced periodontal bone loss, to increase proprioception, improve retention, and stability of periodontal prosthesis. The secondary purpose is to establish an environment conductive to professional and personal plaque control during the maintenance phase. The design of a CSCTD design seems to be a better choice as compared to the clasped removable partial denture.

Clinical evaluations of the CSCTD with magnetic attachment restoration revealed a remarkable improvement around periodontal tissue of the inner crowns and intra-furcation and furcation roof of \#17 after molar root separation (Fig 1) The probing depth, clinical attachment level, and periodontal health of most abutments remained within normal ranges ( $\leqq 3 \mathrm{~mm}$ ) after periodontal and prosthetic therapies using the CSCTD with magnetic attachment.

The present case report revealed that the effectiveness of plaque control and remarkable periodontal healing as compared to the \#17 with Class II \& III molar FI without root separation. Similar findings were also noted for the scores of GI, PD, and CAL.

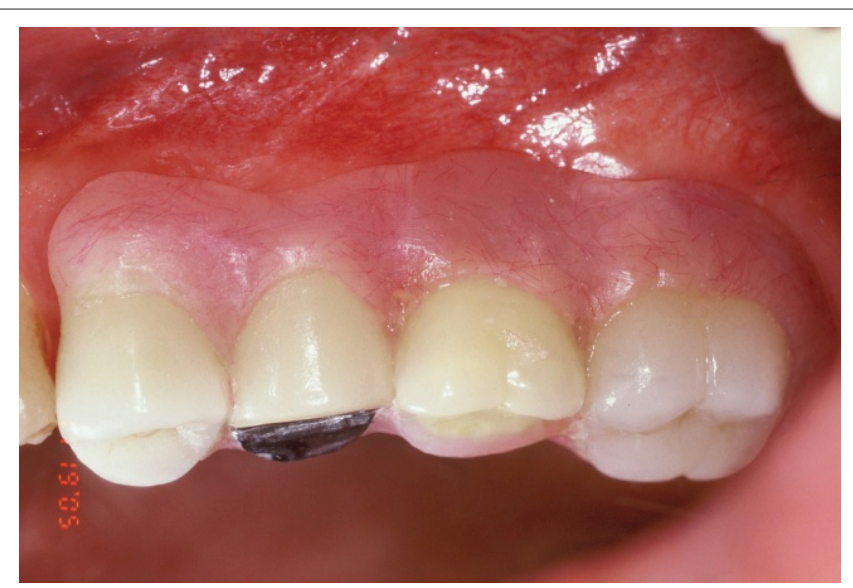

Figure 3: Buccal surface CSC-telescopic crown with magnetic attachment. (\# 14).

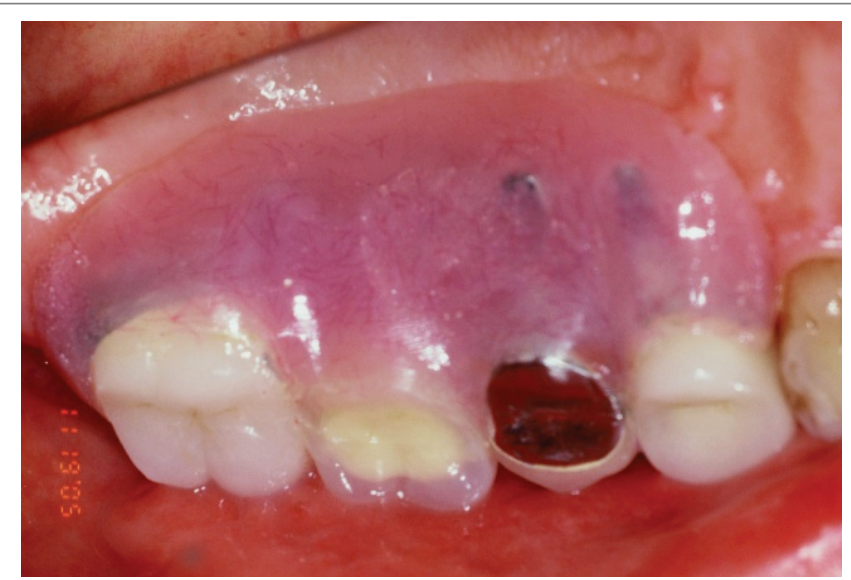

Figure 4: Palatal surface CSC-telescopic crown with magnetic attachment (\#14). 


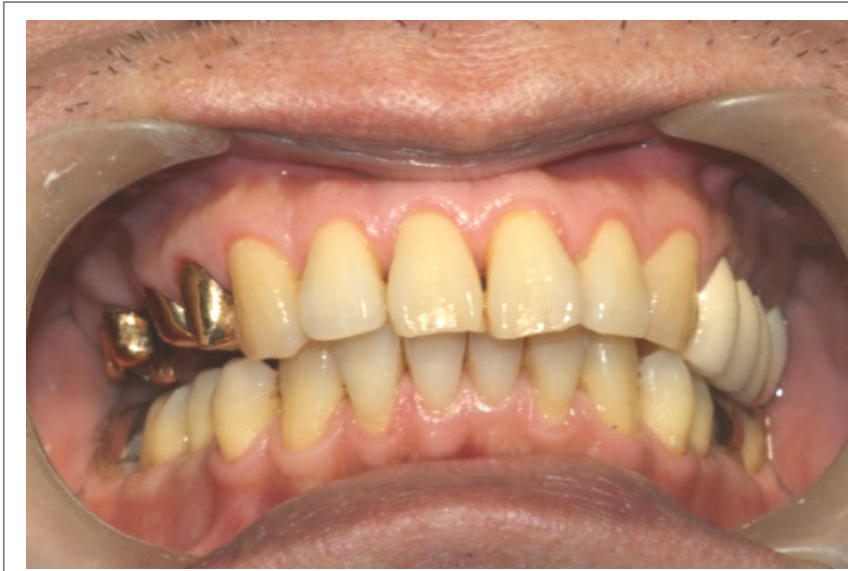

Figure 5: Facial view shows gold inner crowns of \# 14, \#15, and \# 17(2019).

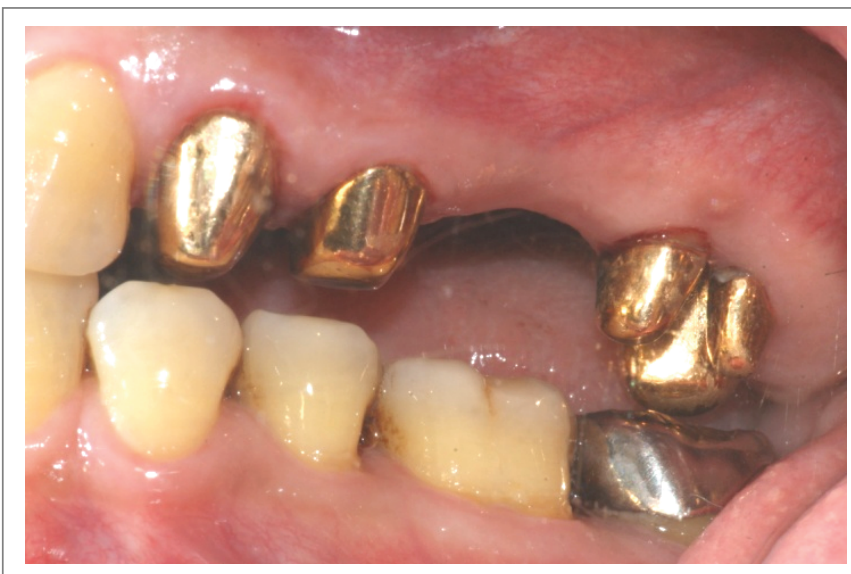

Figure 6: Buccal surface (right posterior) presents the inner crowns $(\# 14, \# 15)$ and three roots separation of \#17.

Muller HP, et al. [3] presented that about $44 \%$ of molars with Class III FI were extracted after periodontal surgery, irrespective of recall visits and sub-gingival scaling. At lesion sites with $\geqq 5 \mathrm{~mm}$ and bleeding on probing, evaluation visits were carried out every 2 to 3 months for professional prophylaxis. They concluded that the decision for the selecting a treatment modality appears to depend on the degree of molar FI as well as tooth type.

Some investigators have reported that the limited and less predictable success of the treatment modality of Classes II and III maxillary furcation defects seems to depend essentially on the class molar FI and root morphology, irrespective of the operator's skill and severity of the periodontitis $[3,4,6,13]$. They concluded that factors such as furcation anatomy and size of the defects between the alveolar bone and tooth and the amount of remaining periodontium facing the defect are also important.

The fact in the current report a remarkably greater improvement in periodontal tissues healing has been obtained following molar root separation and fabrication of CSCTD with magnetic attachment design appeared to be more predictable result than molars without root separation. This finding strongly suggests that this special design of both molar FI with root separation and CSCTD with magnetic attachment appears to be a valuable technique in treating the molar affected with classes II and/or III FI.

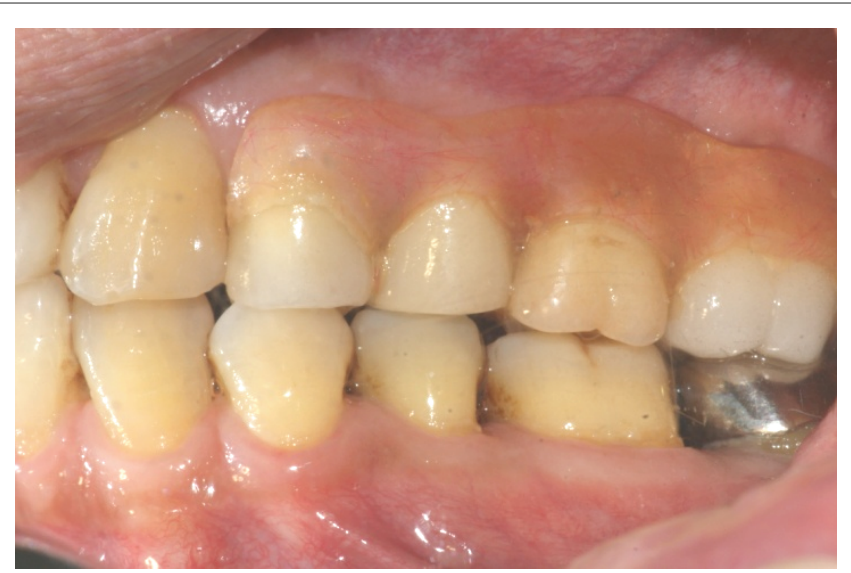

Figure 7: Posterior buccal surface presents the outer telescopic crowns of \#14, \#15) and \#17after13 years (2019).

Majority of the documented reports related to the successful treatment of CSCTD are case reports [11,12]. In 1999, Hou GL, et al. [13] presented a case series study associated with long-term treatment of molar furcation involvement using root separation and a CSCTD for $6.7 \pm 1.9$ years. More recently, Hou GL, et al. $[16,17]$ also documented two long-term studies showed very excellent and remarkable improvement of molars with advanced FI in treating severe advanced periodontitis with secondary occlusal trauma for 5.1-39 years. Results showed that it got not only the effective and prominent in the periodontal soft and hard tissues healing, but also, increase the survival rates of molars with classes II and III FIs using the Sandwich's technique.

The long-term clinical evaluation of present report concluded that the technique in treating the molar with FI using the CSCTD combined magnetic attachment design seems to be a valuable and effective method for dental clinicians.

\section{References}

1. Hirschfeld L, Wasserman B (1978) A long term survey of bone loss in 600 treated periodontal patients. J Periodontol 49: 225-237.

2. Ross Fl, Thompson RH (1978) A long-term study of root retention in the treatment of maxillary molars with furcation involvement. J Periodontol 49: 238-244.

3. Muller HP, Eger T, Lange DE (1995) Management of furcationinvolved teeth. A retrospective analysis. J Clin Periodont 22: 911917.

4. Hou GL, Chen SF, Tsai CC (1996) Furcation entrance dimension. Divergent, angle, and length of CEJ to furcation entrance related to periodontal therapy. Kaohsiung J Med Sci 12: 705-715.

5. Hamp SE, Nyman S, Lindhe J (1975) Periodontal treatment of multirooted teeth. Results afyer 5 years. Clin J Periodont 2: 126-135.

6. Carneval G, Febo GD, Tonell MP, Marin C, Fuzzi M (1991) A retrospective analysis of the periodontal-prosthetic treatment of molars with inter-radicular lesions. Inter J Perio Restor Dent 11: 189-205.

7. Langer B, Stein SD, Wagenberg B (1981) An evaluation of root resection. A ten-year study. J Periodontol 52: 719-722.

8. Wagenberg J (1980) The furcation problem. Etiology, pathogenesis, diagnosis, therapy, and prognosis. J Clin Periodont 7: 73-95. 
9. Majzoub Z, Kom S (1992) Tooth morphology following root resection procedures in maxillary first molars. J Periodontol 63: 290-296.

10. Mardam-Bay W, Majzoub Z, Kon S (1994) Anatomic considerations in the etiology and management of maxillary and mandibular molars with furcation involvements. Int J Periodontics Restorative Dent 14: 399-409.

11. Yalisove IL (1966) Crown and sleeve-coping retainers for removal partial dentures. J Prosthet Dent 16: 1069-1085.

12. Hou GL (1994) A longitudinal study of periodontal and prosthetic therapy in severely advanced periodontitis. Chinese Dent J 14: 16.

13. Hou GL, Tsai CC, Weisgold AS (1999) Treatment of molar furcation involvement using root separation and a crown and sleeve-coping telescopic denture. A longitudinal study. J Periodontol 70: 1098-1109.
14. Loe H, Silness J (1963) Periodontal disease in pregnancy. I. Prevalence and severity. Acta Odontol Scand 21: 553- 551.

15. Silness J, Loe H (1964) Periodontal disease and pregnancy. II. Correlation between oral hygiene and periodontal condition. Acta Odontol Scand 22: 121-135.

16. Hou GL, Hou LT (2019) Therapeutic outcomes using the Sandwich's technique in treating severe advanced periodontitis with secondary occlusal trauma: A long-term study for 5.1-39 years. Intern J Dent and Oral Health 5.

17. Hou GL (2020) Survival rates of CSC Telescopic Abutments of severe advanced periodontitis with secondary occlusal traumatism using periodontal prosthetic therapies. A long- term study of case series for 5.1-19.3 years. Advances Dent Oral Health. 